Recherches en didactique des langues et des cultures

Les cahiers de l'Acedle

15-2 | 2018

Quelles médiations en didactique des langues et des cultures?

\title{
Parler ou ne rien dire : La prise de parole en classe d'anglais Lansad
}

\section{Elisabeth Crosnier et Nicole Décuré}

\section{(2) OpenEdition}

Journals

Édition électronique

URL : http://journals.openedition.org/rdlc/3062

DOI : $10.4000 /$ rdlc.3062

ISSN : 1958-5772

Éditeur

ACEDLE

Référence électronique

Elisabeth Crosnier et Nicole Décuré, «Parler ou ne rien dire : La prise de parole en classe d'anglais Lansad », Recherches en didactique des langues et des cultures [En ligne], 15-2 | 2018, mis en ligne le 02 juin 2018, consulté le 30 avril 2019. URL : http://journals.openedition.org/rdlc/3062 ; DOI : 10.4000/ rdlc.3062

Ce document a été généré automatiquement le 30 avril 2019

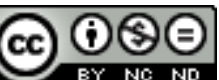

Recherches en didactique des langues et des cultures is licensed under a Creative Commons AttributionNonCommercial-NoDerivatives 4.0 International License 


\title{
Parler ou ne rien dire : La prise de parole en classe d'anglais Lansad
}

\author{
Elisabeth Crosnier et Nicole Décuré
}

\section{Introduction}

1 L'expression orale en langue vivante, largement préconisée en Europe (CECRL), est devenue en France une préoccupation majeure pour les premier et second cycles, et les compétences orales des jeunes apprenants font l'objet d'évaluations régulières. Toutefois, dans l'enseignement supérieur, elles sont développées au niveau de chaque établissement, selon des décisions individuelles ou collectives locales. Dans notre université de sciences et santé, Toulouse 3, la politique affichée pour les langues (l'anglais en fait) est de privilégier au maximum l'oral - compétence par ailleurs très demandée de la part des étudiant.e.s. Comme il est admis depuis quelques décennies que l'on apprend à parler en parlant, il s'ensuit que la pratique de l'oral doit être une priorité dans la classe de langue.

2 Tout.e enseignant.e d'anglais du secteur Lansad sait que « faire parler la langue » est à la fois la chose la plus nécessaire dans une salle de classe (Pogranova et al., $2017: 101)$ et la moins facile. Tout.e étudiant.e d'anglais en Lansad sait que parler est la chose la plus désirable, la plus indispensable - puisque dans le monde actuel l'oral est omniprésent. Enfin, de nombreuses études, trop nombreuses pour les citer ici, montrent que c'est l'activité la plus anxiogène. À partir de cette problématique, nous pouvons nous demander s'il est possible de créer des conditions pour qu'une personne n'ait pas d'autre option que celle de parler.

3 L'interaction orale est inéluctable : elle est dans l'instant puisqu'il faut comprendre tout de suite et répondre sur le champ et que les partenaires n'ont pas le temps de relire des phrases ni de peaufiner un texte; ils/elles sont soumis.e.s à la pression du temps. Cette immédiateté, cette rapidité d'exécution, sans laquelle on se sent incompétent.e, sont à l'origine même des problèmes. Lire, écrire ou écouter peut se faire à loisir, chez soi, alors que pour parler avec des partenaires en contact direct, sans l'intermédiaire d'écrans qui 
limitent le contact, la classe reste quasiment le seul endroit possible pour cette pratique. L'expression orale constitue un champ de recherche à la fois immense et complexe, qui a fait l'objet de multiples investigations, selon des perspectives diverses (Dörnyei \& Kormos, 1998 : 349-385 ; Germain \& Netten, 2005 ; Hilton, 2011 ; Moussa, 2010 ; Osboe et al., 2007 ; Park \& Lee, 2007; Yoshimura \& MacWhinney, 2007; Weissheimer \& Mota, 2011). Tous ces travaux permettent de comprendre les processus et les mécanismes mis en œuvre à l'oral et visent à améliorer les compétences des apprenant.e.s. La qualité de la langue, la phonétique, les procédures de correction, l'aisance et le débit sont au cœur des préoccupations en matière d'acquisition d'une L2 à l'oral. Ils attestent de la richesse du domaine mais également de la difficulté à en cerner tous les aspects. L'envie de parler en classe ou encore l'initiative de la prise de parole devant le groupe paraissent avoir été peu explorées (Tighilet, 2016) et ce d'autant moins dans l'environnement Lansad. Cette analyse est difficile à faire pour des raisons de perception et de subjectivité. Les enregistrements audio et vidéo, s'ils sont plus objectifs, ne peuvent prendre en compte la réalité globale de la classe (Carvalho, 2015). Des méthodes encore plus invasives risqueraient de faire perdre la spontanéité des étudiants s'ils/elles se sentaient trop ouvertement observé.e.s (Nguyen, 2014).

Il semble pertinent, actuellement, de mener des investigations sur ces thèmes. Les relations entre la nature des activités et les sujets traités, les conditions de prise de parole et la perception qu'ont les étudiant.e.s de l'expression orale constituent des paramètres de première importance, avant même de considérer la qualité de cette expression orale. Nous émettons l'hypothèse que certaines activités, dans certaines conditions, seraient inhibitrices alors que d'autres peuvent être stimulantes et de nature à donner aux étudiant.e.s l'envie de surmonter les obstacles pour prendre la parole. Dans ce cas, le désir de parler inciterait les apprenant.e.s à faire abstraction des considérations matérielles intellectuelles, psychologiques ou culturelles ou à les surmonter.

5 L'objectif de notre travail a été de repérer les facteurs susceptibles de déclencher la parole afin que les enseignant.e.s privilégient ces actions. Nous ne nous sommes pas intéressées à ce qui était dit (contenu du message), ni à sa qualité (correction, fluidité), ni au registre (formel, informel) mais simplement à l'essence de toute communication orale (Gan, 2012: 43-59), ce qui intervient en amont: l'intention de parler, comme le signale le modèle de Levelt (Dörnyei \& Kormos, 1998: 351-352) suivie ou non de son exécution, l'activité locutoire (Austin, 1962), le fait de dire des mots dans une langue étrangère, mots qui forment ou non des phrases, un discours cohérent ou non, qui impliquent ou non une interaction avec une/des autre.s personne.s. En raison de l'immense variabilité des situations, nous doutons qu'un modèle puisse être appliqué. Toutefois, nous essaierons de proposer un cadre optimal pour la pratique de l'expression orale.

\section{Contexte de l'étude}

6 Depuis une vingtaine d'années, des enquêtes à grande échelle à l'Université Toulouse 3 ont été effectuées auprès de nos étudiant.e.s sur des sujets tels que la motivation (Décuré, Lavinal \& Blois, 2005), l'évaluation de leur année ou encore les activités orales mêmes (Décuré, 2000). Elles nous ont fourni des données sur la place occupée par l'oral et sa pratique. De cette perspective diachronique il ressort, de façon constante, que l'oral est une activité langagière à la fois désirable et source de difficulté (impossibilité de 
s'exprimer) et d'anxiété (timidité, lathophobie), essentiellement en raison d'un manque de pratique.

Lors de nos réunions pédagogiques, il est apparu que l'appréciation de ce qui constitue une activité orale variait considérablement d'une personne à l'autre. Nous avons alors voulu savoir ce qui se passait réellement dans les classes en interrogeant nos propres étudiant.e.s sur leur activité en classe. Plusieurs enseignantes se sont impliquées dans ce travail de recherche mais seules les deux auteures de cet article ont mené l'expérience à son terme. Les activités orales prises en considération dans ces deux contextes sont, pour la plupart, non préparées hors de la classe (sauf exposés) et donc spontanées et sur la base du volontariat (voir infra). Deux préoccupations dominent : réduire autant que possible le temps de parole de l'enseignante afin de laisser la parole aux étudiant.e.s (centration sur l'apprenant.e) ; et se rapprocher le plus possible de situations authentiques par le biais de tâches (task-based learning) sans toutefois entretenir l'illusion que cela soit vraiment possible dans un environnement universitaire. Pour Bourguignon, qui parle d'approche "communic'actionnelle", "la tâche communicative [...] reste le pivot de l'enseignementapprentissage des langues » (Bourguignon, $2011: 52$ ).

\section{Méthode}

8 Afin de traiter au mieux notre problématique sur la production orale de nos étudiant.e.s dans une université scientifique, nous leur avons soumis des questionnaires anonymes visant à obtenir des données sur leur ressenti, donc leurs représentations (cf. Annexe 1). De leur côté, les enseignantes ont fait un descriptif factuel de chaque séance dans un carnet de bord (cf. Annexe 2) afin de vérifier l'influence éventuelle de ces facteurs sur la prise de parole. Certains sont d'ordre matériel, imposés par l'institution, sur lesquels les enseignant.e.s de langue ne peuvent généralement pas intervenir: l'effectif et la composition des groupes, aléatoire ou arbitraire, les créneaux horaires et la configuration de la salle. C'est également le cas pour le nombre d'années d'étude, l'anglais en tant que langue imposée, plus ou moins explicitement, la réserve face à une nouvelle enseignante (qui, généralement, change à chaque semestre), surtout au début du module, ou les difficultés liées à l'anglais pour les sciences et à visée professionnelle. D'autres paramètres peuvent être infléchis dans une certaine mesure : la confiance et l'estime de soi/des autres entretenues précédemment, la crainte du jugement et de l'échec, la perte de la face, souvent induites par les expériences précédentes en cours de langues, les inhibitions et introversions (Park \& Lee, 2007 : 198-199). Certaines de ces variables ont été intégrées dans le questionnaire.

\section{L'enquête}

Le public

Tableau 1 - Présentation des groupes

\begin{tabular}{|l|l|l|l|l|l|l|l|l|l|}
\hline & $\begin{array}{l}\text { Code } \\
\text { gr. }\end{array}$ & $\begin{array}{l}\mathrm{Nb} \\
\text { inscrits }\end{array}$ & $\begin{array}{l}\text { Moyenne } \\
\text { présences }\end{array}$ & Nb. F/G & $\begin{array}{l}\text { Nb } \\
\text { sem. }\end{array}$ & $\begin{array}{l}\text { Durée } \\
\text { séances }\end{array}$ & Horaire & Niveau & Anglais \\
\hline L3 SID & A1 & 19 & 16 & $6 \mathrm{~F} / 13 \mathrm{G}$ & 10 & $2 \mathrm{~h} 00$ & $13 \mathrm{~h}-15 \mathrm{~h}$ & A1 & général \\
\hline
\end{tabular}




\begin{tabular}{|c|c|c|c|c|c|c|c|c|c|}
\hline M1 SID & A2 & 14 & 12 & $4 \mathrm{~F} / 10 \mathrm{G}$ & 10 & $2 \mathrm{~h} 00$ & $15 \mathrm{~h}-17 \mathrm{~h}$ & B1 & \\
\hline $\begin{array}{l}\text { M1 } \\
\text { Informatique }\end{array}$ & A3 & 24 & 16 & $2 \mathrm{~F} / 22 \mathrm{G}$ & 11 & $2 \mathrm{~h} 00$ & $16 \mathrm{~h}-18 \mathrm{~h}$ & A2-B2 & \\
\hline $\begin{array}{l}\text { M2 } \\
\text { Pharmacie }\end{array}$ & $\mathrm{A} 4$ & 22 & 19 & $21 \mathrm{~F} / 1 \mathrm{G}$ & 8 & $2 \mathrm{~h} 30$ & $17 \mathrm{~h} 30-20 \mathrm{~h}$ & A2-B1 & \\
\hline L3 BCP & B1 & 18 & 14 & $10 \mathrm{~F} / 8 \mathrm{G}$ & 8 & $2 \mathrm{~h} 00$ & $15 \mathrm{~h} 45-17 \mathrm{~h} 45$ & $\mathrm{~A} 1-\mathrm{C} 1$ & \multirow{3}{*}{$\begin{array}{l}\text { scientifique } \\
\text { et } \\
\text { professionnel }\end{array}$} \\
\hline L3 BCP & B2 & 15 & 13 & $8 F / 7 G$ & 7 & $2 \mathrm{~h} 00$ & $18 \mathrm{~h}-20 \mathrm{~h}$ & $\mathrm{~A} 1-\mathrm{C} 1$ & \\
\hline M2 MSE & B3 & 15 & 12 & $8 \mathrm{~F} / 7 \mathrm{G}$ & 7 & $2 \mathrm{~h} 00$ & $13 \mathrm{~h} 30-15 \mathrm{~h} 30$ & $\mathrm{~A} 1-\mathrm{C} 1$ & \\
\hline Total & & 127 & & $59 \mathrm{~F} / 68 \mathrm{G}$ & & & & & \\
\hline
\end{tabular}

9 Tous les groupes ${ }^{1}$, sauf deux, sont de niveau hétérogène, un même groupe pouvant comprendre à la fois des étudiant.e.s de niveau A1, des bilingues et même des anglophones. Ils/elles sont âgé.e.s de 20 à 25 ans avec, dans la plupart des cas, de sept à neuf années d'anglais à leur actif. Les sept groupes ont été sollicités pendant plusieurs semaines (entre sept et dix) d'un semestre, selon les emplois du temps et les disponibilités. Les épreuves orales de contrôle continu ont été incluses dans les activités prises en compte dans les questionnaires.

\section{Le questionnaire}

10 Le questionnaire a d'abord été administré à des groupes identiques à ceux du Tableau 1, sous la forme de pré-test, à partir de nos précédentes enquêtes (voir section 2). L'analyse des résultats nous a conduites à le modifier et à ajouter une rubrique concernant le degré de satisfaction pour une approche moins factuelle, plus qualitative, plus globale. Nous avons dû préciser, dès la première séance, que nous considérions l'oral comme étant l'action de dire quelque chose en anglais car ce pré-test avait révélé que de nombreux/ses étudiant.e.s avaient une définition de l'oral restrictive, comme répondre aux questions des enseignant.e.s (Behra \& Macaire, 2017 : 125-126) ou faire un exposé devant la classe, et excluaient les autres formes d'intervention orale (interactions entre étudiant.e.s, interventions spontanées, etc.).

11 Nous avons distribué le questionnaire à la fin de chaque séance pendant plusieurs semaines (de 7 à 11 selon les groupes) afin de recueillir des données. Pour que les réponses soient spontanées et données rapidement, les questions fermées ont été privilégiées. Elles ont été regroupées dans quatre catégories: le temps de parole de chacun.e (intensité de la pratique); les partenaires de la communication (centrée sur l'apprenant.e ou sur l'enseignant.e); les difficultés ressenties (les obstacles à la communication); l'impression générale de (non)satisfaction par rapport à la séance qui s'achevait, traduisant l'intérêt pour les activités et la motivation.

12 Les questions fermées possèdent l'avantage de fournir des informations quantitatives pouvant être traitées aisément. Celles-ci permettent également d'éviter en partie l'écueil de la subjectivité du fait qu'elles sont récentes, donc non déformées par la mémoire. Cependant, bien qu'elles soient forcément subjectives et discutables car déclaratives 
(Osboe et al., 2007: 3-5), elles sont susceptibles de fournir des indications sur les comportements des étudiant.e.s et leur interprétation de ces comportements.

Cette procédure, testée lors d'un premier semestre en 2014-2015, a été affinée en 2015-2016 et, à de rares exceptions près, la tâche a été accomplie consciencieusement et la recherche prise au sérieux par les étudiant.e.s (cf. Annexe 3).

\section{Le carnet de bord}

Pour compenser la part de subjectivité des réponses, nous avons établi un carnet de bord dans lequel chaque enseignante répertoriait les activités effectuées au cours d'une séance avec leur durée et leur intitulé. Il est indissociable des questionnaires. Pour chaque activité étaient notés la topographie de la classe (en rangées, en U, en îlots), le type de prise de parole (préparée ou non), le type d'interaction (guidée ou libre, classe entière ou petit groupe), la tâche à effectuer à partir d'un support ou non, individuellement ou en groupe. Ce système permet de confronter le contenu des deux documents afin de croiser les données, de dresser un panorama des activités de production orale des étudiant.e.s mais aussi de détecter les facteurs encourageants ou inhibiteurs. Les types d'activités de chaque séance pouvant être différents à chaque fois, les variations devaient nous aider à repérer les activités favorisant ou non l'oral pour un groupe donné et pour l'ensemble des groupes, et les examiner en tenant compte des variations de niveau, d'âge, de formation, etc.

\section{Les activités}

Pour comprendre les performances des étudiant.e.s, il est utile de présenter une classification des activités. Si on s'inspire des spoken genres de Figuereido \& Bota (2009 : 102 ; 104), nos interventions sont de type descriptif et argumentatif et elles incluent de la résolution de problèmes, des jeux et des simulations, du small talk et des présentations. Préparées et non préparées, elles impliquent des registres différents mais toujours de l'interaction (Gan, 2012: 46). Les sujets des présentations préparées à la maison sont toujours choisis par les étudiant.e.s.

Nous avons répertorié une quarantaine d'activités différentes (cf. Annexe 3). Une grande part de créativité a été nécessaire pour les adapter aux besoins spécifiques de chaque classe, soit à partir de tâches et de cadres existants, soit créées ex-nihilo.

Elles se divisent en six types : les jeux, les débats, les exposés/présentations, la lecture à voix haute/prononciation, la reformulation, l'improvisation. La moitié est de nature guidée ou contrainte, l'autre moitié relève de l'improvisation. Cinq activités sur six se font à l'aide d'un support (qui peut aller d'un mot à un document long). Une légère majorité d'activités se pratique en petits groupes (de deux à six) sans préparation. Très peu d'activités sont préparées en classe, même rapidement, ou à la maison (exposés), une grande majorité des activités étant réalisée de façon spontanée et improvisée.

Notre premier souci, pour favoriser l'expression orale, a été de réorganiser l'espace-classe de façon à réduire les obstacles à l'interaction entre étudiant.e.s. 


\section{Résultats de l'enquête par questionnaire}

Nous avons exploité 808 questionnaires, chaque questionnaire (Annexe 1) correspondant à une séance pour un.e étudiant.e. Chaque séance ayant comporté en moyenne trois activités différentes, une évaluation fine pour chacune est impossible et, par conséquent, les chiffres se réfèrent à une impression globale par séance.

\section{Le temps de parole (rubrique 1)}

20 Nous sommes conscientes que l'évaluation de la durée de prise de parole dépend de la perception du temps de chaque individu (Droit-Volet, 2005), de sa conception du temps qui passe et de son ressenti au cours de l'activité en fonction d'un certain nombre de paramètres : l'intérêt que suscite la tâche à accomplir, l'envie d'intervenir, le débit de parole, le niveau d'anglais, la maîtrise du sujet et de la langue, la mobilisation d'idées, les différences culturelles. À ceci s'ajoute un niveau collectif: les réactions au sein de la classe et les imprévus de l'évolution des situations. Seules les interventions qui impliquent un chronométrage strict, comme les présentations en temps limité, peuvent être évaluées objectivement. Les réponses à cette rubrique étaient nécessairement subjectives lorsqu'elles visaient les activités incluant spontanéité et improvisation. Nous devrons alors les interpréter avec grande prudence.

21 La moitié des étudiant.e.s déclare un temps de parole supérieur à cinq minutes par séance, ce qui montre que l'objectif de favoriser la parole est en partie atteint. Il y a cependant des disparités entre les groupes.

Les groupes $\mathrm{A}$, répartis par tables de quatre, ont des cours d'anglais général dont le but unique est l'expression orale. Dans trois d'entre eux, If (parler plus de 15 minutes) représente entre $35 \%$ et $47 \%$; il est rare que quelqu'un déclare ne pas parler ou parler moins d'une minute. Dans le groupe A1, en revanche, cela s'est produit régulièrement : du fait que l'interaction repose sur l'envie de communiquer, rien n'oblige certain.e.s à s'exprimer puisque l'enseignante n'est pas là en permanence pour les y inciter et les camarades n'insistent guère.

Dans les groupes $\mathrm{B}$, où toutes les activités sont partagées avec la classe entière, même celles qui ont été effectuées en petits groupes au préalable, le taux de 1f est faible (de $2 \%$ à $7 \%$ ). Certaines activités liées à l'anglais scientifique (lecture, écriture) contribuent à réduire le temps consacré à l'expression orale, d'autant plus que le manque de compétences scientifiques pointues de l'enseignante inhibe sa créativité et réduit le champ des possibles, surtout pour la conception d'activités spécifiques. Les tentatives de faire créer des tâches autres que les exposés par les étudiant.e.s n'ont pas été probantes.

On peut cependant se demander si, dans le travail en petits groupes, les étudiants parlent vraiment dans la langue cible puisque l'on sait, et les commentaires libres des étudiants à la fin du questionnaire le confirment, que dans une classe endolingue, la tentation d'utiliser la langue commune (ici le français) est grande car parler dans une langue étrangère n'est pas naturel et certain.e.s ne peuvent s'y résoudre (Gan, 2012: 46-47; Osboe et al., 2007:5). Ceci est surtout vrai dans le cadre de la préparation à une tâche. Mais, quand on rentre directement dans celle-ci, que ce soit un jeu ou un débat, cette difficulté tend à s'estomper. 


\section{Les interlocuteurs/trices (rubrique 2)} avons constaté quelques incohérences de la part des étudiant.e.s comme, par exemple, déclarer ne pas avoir parlé avec leurs camarades alors qu'ils/elles se sont adressé.e.s au groupe ou à une partie du groupe (Behra \& Macaire, 2017 : 125-126) : trop habitué.e.s à répondre aux sollicitations de l'enseignante, ils/elles ne réalisent même plus qu'il faut prendre en compte, les interactions avec leurs partenaires dans la classe. Ces incohérences sont impossibles à traiter bien qu'elles soient révélatrices du conditionnement des étudiant.e.s. Nous constatons toutefois que la parole circule et pas seulement avec l'enseignante.

\section{Les difficultés (rubrique 3 )}

La troisième partie du questionnaire, portant sur les difficultés rencontrées lors de la prise de parole, regroupe des données de type linguistique, mental, psycho-affectif. Ces questions paraissent moins subjectives mais il faut à nouveau s'interroger sur l'écart entre ce qui est perçu et la réalité. Par exemple, des hésitations interprétées comme étant dues à des faiblesses en matière de vocabulaire peuvent avoir d'autres causes.

Le manque de vocabulaire arrive en tête dans six groupes sur sept. Les difficultés de prononciation et de grammaire sont à égalité. Un petit tiers est à l'aise et dit ne pas percevoir d'obstacles. Là encore, il y a des variations entre les groupes.

\section{Pas de difficultés}

Un groupe (B1) se distingue des autres dans la mesure où presque la moitié pense ne pas avoir de problèmes particuliers pour s'exprimer en anglais, bien que le niveau des étudiant.e.s varie de $\mathrm{A} 2 \mathrm{à} \mathrm{C} 1$. Rien, cependant, ne permet d'avancer une explication à ce phénomène. En revanche, dans le groupe A4, 10 \% seulement déclarent ne pas avoir eu de difficultés. Ceci peut s'expliquer par l'effort intense de prise de parole demandé : séances longues de $2 \mathrm{~h} 30$, travail uniquement d'interaction orale en petits groupes.

\section{Difficultés de vocabulaire}

Les groupes B éprouvent moins de difficultés liées au vocabulaire que les groupes A. On peut avancer ici une explication par la différence de type de lexique: la terminologie scientifique et para-scientifique est limitée, connue ou reconnaissable (termes transparents), ce qui n'est pas le cas pour l'anglais général qui couvre de multiples champs. Dans le groupe A1, en raison du faible niveau général (A2), à la fois réel et ressenti comme un échec par les étudiant.e.s, le manque de vocabulaire apparaît comme une explication facile et convenue.

\section{Difficultés de prononciation}

La prononciation n'apparaît pas comme un problème, d'autant moins que nous favorisons la fluidité et l'aisance dans l'interaction plutôt que la justesse. Les deux groupes le plus en difficulté sont le groupe très faible (A1) et le groupe qui devait parler le plus (A4).

Recherches en didactique des langues et des cultures, 15-2 | 2018 


\section{Difficultés de grammaire}

31 Les mêmes remarques s'appliquent à la grammaire. L'oral spontané, d'ailleurs, est moins exigeant en matière de complexité des structures.

\section{Difficultés de compréhension}

Il y a apparemment peu de difficultés à comprendre les autres, sauf dans le groupe de niveau A1 puisque la communication se fait entre personnes dont les difficultés sont globalement semblables et que les enseignantes font le nécessaire pour être comprises. Les étudiant.e.s n'ont pas pris en compte la compréhension de l'oral à partir de documents audio car les résultats sont les mêmes pour les deux enseignantes alors que l'une a fait cette activité systématiquement chaque semaine et l'autre très rarement.

\section{Manque d'idées}

Dans les discussions informelles, le manque d'idées comme raison de mutisme est souvent avancé par les étudiant.e.s et c'est pourquoi nous avions inclus ce paramètre dans le questionnaire. L'enquête ne confirme cependant pas que ce soit un problème majeur puisque moins d'un cinquième des étudiant.e.s le mentionne alors que les activités de débat, d'expression d'idées ou de connaissances sont nombreuses.

\section{Timidité, angoisse, inhibition - Manque de motivation / d'envie / découragement}

Les facteurs d'ordre psycho-affectif sont souvent cités comme responsables d'inhibitions dans les apprentissages, des langues en particulier (Arnold, 2006). Park \& Lee (2007 : 199) affirment qu'une atmosphère détendue grâce à une mise en confiance ne suffit pas toujours à libérer la parole. Cependant, dans notre cohorte, il est rassurant de constater que les étudiant.e.s ne semblent pas souffrir de timidité ni d'angoisse : ils/elles ne sont pas inhibé.e.s pour s'exprimer. De plus, n'avouant pas un manque de motivation, parler ne présente donc pas d'obstacle conscient.

\section{Le degré de satisfaction (rubrique 4)}

Pour la quatrième partie du questionnaire, portant sur la (non)satisfaction par rapport au temps de parole déclaré (rubrique 1), c'est directement le ressenti que nous avons cherché à connaître, le sentiment de satisfaction.

Si on regarde le temps de parole de celles et ceux qui déclarent avoir suffisamment parlé (la moitié), on voit que l'horizon d'attente dans certains groupes n'est pas très élevé. Il se peut cependant que, par rapport à leurs expériences antérieures, parler même deux minutes leur semble long. ${ }^{2}$ Quant à ceux-celles qui pensent avoir beaucoup parlé, ils/elles ne représentent que $15 \%$ en moyenne mais cela peut aller de $0 \%$ à $27 \%$, ce qui constitue une différence importante.

Dans certains groupes (A2 et A4), le taux de satisfaction coïncide avec un temps de parole de plus de cinq minutes. Dans un autre (A1), il semble y avoir contradiction entre les deux éléments car moins de la moitié des étudiant.e.s parle plus de cinq minutes mais quatre sur cinq sont satisfait.e.s, ce qui n'est pas surprenant dans un groupe de niveau A1 pour 
lesquel.le.s parler est une épreuve, voire un calvaire. Dans ce groupe, c'est leur niveau qui explique leur score. Ils/Elles débutent fréquemment avec un sentiment d'échec dans leur cursus de trois ans. L'expérience (les relevés de notes au fil des ans) montre que les résultats de ces étudiant.e.s, une fois entrés en M1 l'année suivante, sont bien meilleurs (ces ex-L3 eux aussi parlaient peu en première année d'IUP). L'insistance sur l'oral semble donc bien avoir une influence bénéfique, non pas à court terme pendant la même année, mais à plus long terme. En effet, d'une année à l'autre, on ne peut guère espérer voir une progression de niveau significative car 24 ou 48 heures d'exposition à la langue ne suffisent pas (Snauwaert, 2015). Mais les étudiant.e.s gagnent en confiance (en maturité aussi sans doute) et sont mieux à même de mobiliser leurs ressources langagières.

\section{Le questionnaire de bilan}

38 Un questionnaire de bilan (cf. Annexe 4) a été distribué lors de la dernière séance dans le but d'obtenir quelques précisions. Le temps de parole n'étant plus quantifié, il ne reste que la perception globale qui tend à contredire l'évaluation semaine après semaine. En effet, la presque totalité des répondant.e.s s'estime satisfaite d'avoir beaucoup parlé. Il est difficile de savoir ce que représente le terme "beaucoup» chez les étudiant.e.s qui n'avaient pas le choix de répondre «suffisamment " parce que nous avions sciemment éliminé ce terme trop neutre. Il y a également l'image de soi que l'on veut renvoyer, malgré l'anonymat, et que la plupart veulent positive. Rappelons aussi que même s'ils/ elles ont peu parlé, c'est plus que dans leur scolarité antérieure.

Comme dans de nombreux questionnaires de ce type, la rubrique de commentaires libres est très peu renseignée. On trouve essentiellement des regrets par rapport au manque d'exercices de grammaire et/ou de vocabulaire et par rapport au travail effectué en groupes au détriment de la formule classique du tour de parole, phénomène qu'ont rappelé Kern (1995) et Spratt (1999) en référence à d'autres études. Il y a aussi des remerciements pour cette approche orale du cours, avec une mention spéciale pour certaines activités telles que les jeux, les débats et les réactions spontanées.

\section{Relation entre résultats des questionnaires et nature des activités}

Si nous émettons l'hypothèse que certaines activités, dans certaines conditions, seraient inhibitrices alors que d'autres peuvent être stimulantes et de nature à donner aux étudiant.e.s l'envie de prendre la parole, nous devons mettre en corrélation des réponses d'étudiant.e.s et les activités réalisées pour voir si, effectivement, ces dernières ont influencé les comportements. Nous avons cherché, dans chaque groupe, les séances qui ont obtenu des scores maximum dans le questionnaire étudiant (temps de parole, absence de difficultés, satisfaction) et les avons confrontées aux activités notées sur le carnet de bord. Nous avons sélectionné quelques résultats particulièrement significatifs.

41 Il est souvent difficile d'interpréter ces données car les variations entre les groupes sont importantes. Cependant, nous pouvons énoncer quelques constantes.

- Le travail à deux ou en petits groupes augmente le temps de parole. Ceci est très visible si l'on compare les groupes A et B (cf. section 4.1.). 
- L'improvisation, la spontanéité, c'est-à-dire les activités non contraintes et non préparées, ne semblent pas être, comme on pourrait le craindre, des facteurs inhibiteurs. Le groupe A4 atteint son maximum de temps de parole dans des séances consacrées uniquement à ce type d'activités. Dans le groupe A3, les deux premières semaines concentrent le maximum de temps de parole en dépit des nombreuses difficultés d'ordre langagier exprimées. Il y a une certaine logique à cela : dans un groupe hétérogène et de niveau A1 - A2, plus on parle, plus il y a d'obstacles. Ceci n'empêche pas un taux élevé de satisfaction, ce qui tend à montrer que la performance leur paraît plus importante que la compétence. Les étudiant.e.s du groupe B3 annoncent un temps de parole plus élevé et sont satisfait.e.s d'avoir assez/ beaucoup parlé lors d'une séance d'improvisation complète de remue-méninges sur un thème qui ne leur était pourtant pas familier et malgré des problèmes de vocabulaire. C'est également vrai, de manière plus relative, pour une séance du groupe B1 entièrement consacrée à des activités d'improvisation en anglais général, malgré des difficultés linguistiques plus marquées. Nous parvenons également à cette conclusion, pour les activités contraintes mais non préparées, telles que toutes les activités ludiques. Dans tous ces cas, les obstacles pour trouver les mots spontanément sont réels mais n'inhibent pas la prise de parole.

- Les débats qui sont intégrés à une autre activité ne fournissent pas d'informations en ce qui concerne le temps de parole pour les groupes B. Pour le groupe A4 où cette activité a occupé une séance (semaine 9) à part égale avec un jeu de rôles, le temps de parole est le plus élevé du semestre et le taux de satisfaction de $100 \%$. Les difficultés exprimées sont de tous ordres et atteignent le deuxième taux du semestre mais on voit là encore que la difficulté n'empêche pas la parole de circuler si les activités sont motivantes.

- Les exposés sont un facteur négatif en matière de temps de parole, sauf pour la personne qui expose. Le groupe A3 commence le semestre avec des taux de participation assez hauts, qui s'effondrent la semaine où cette activité commence. Il y avait là une contrainte institutionnelle incontournable avec un cadre pédagogique défini. D’après les données recueillies, notamment pour le groupe B3, les étudiant.e.s ne semblent pas prendre en considération le temps imparti à la discussion qui suit les présentations scientifiques, lequel est difficilement mesurable. On note que, lors des présentations, les difficultés linguistiques ne sont pas toujours proportionnelles au temps de parole mais parfois relatives au type de sujet. Par exemple, elles sont supérieures lors des présentations d'entreprises comparées aux descriptions de procédés scientifiques pour le groupe B3, malgré des conditions identiques.

- Les activités concernant la lecture ou l'écriture, ne favorisent pas l'oral. Ceci explique le différentiel entre les groupes A et B, les premiers les pratiquant rarement, les seconds régulièrement (lecture de documents scientifiques).

- Toutes les activités où la spontanéité est de mise, qu'elles soient contraintes ou non, en anglais scientifique ou général, n'ont pas créé de difficultés liées à l'affect ni bloqué la parole. Elles sont reçues très favorablement par les étudiant.e.s. Les difficultés majeures sont dues au manque de vocabulaire, surtout en anglais général, ce qui est logique. Elles varient selon les thèmes et les tâches plutôt qu'en fonction de leur niveau de difficulté.

\section{Conclusion}

Rappelons d'abord que l'objet de notre étude n'était ni le contenu des cours, ni la qualité de l'expression en anglais, ni la progression mais, le déclenchement de la parole et 
l'activité locutoire à la lumière de trois paramètres : temps de parole, obstacles, degré de satisfaction.

Certains facteurs, relatifs à la constitution et à la mise en place des groupes et qui pourraient être pertinents pour favoriser la prise de parole, nous échappent en grande partie: l'horaire, le ratio hommes/femmes, l'effectif de la classe, l'homogénéité des groupes, leur niveau plus ou moins avancé l'équipement et la configuration des salles de classe, etc. De plus, en raison de la concentration de l'enseignement sur un volume horaire faible, les étudiant.e.s sont privé.e.s des précieux atouts que sont, entre autres, l'habitude de parler ensemble, la création de liens de complicité et de confiance, la diversité des activités. En revanche, lorsque l'on peut exercer sa liberté pédagogique, on a réellement prise sur les formes et les contenus de l'enseignement. Il est possible, par exemple, de choisir les activités et si l'on décide de ne faire que de l'oral, on peut alors vraiment privilégier cette compétence.

L'enquête a souligné les éléments susceptibles d'améliorer la situation : le travail en petits groupes ou binômes, les jeux de toutes sortes, les débats, les activités spontanées. Encore faudrait-il que les salles de classe soient dédiées aux langues, vraiment pensées et aménagées pour l'interaction, et nous ne parlons pas ici de salles multimédia. D'autres moyens, hors cursus, seraient à encourager: communication numérique, cafés des langues, théâtre, stages, voyages. Notons par ailleurs que, selon les spécialités des groupes, la perception et la réception des diverses formes d'expression orale varient. Et c'est bien là qu'importe l'autonomie pédagogique des enseignant.e.s.

Il serait souhaitable de prolonger cette recherche en l'élargissant, par exemple en utilisant comme cohorte des formations entières, soit la même année, soit sur plusieurs années et, pour ce faire, impliquer une équipe large d'enseignant.e.s. L'observation des classes par des personnes extérieures et, dans une certaine mesure, les enregistrements audio et vidéo, pourraient aussi contribuer à enrichir et affiner les données.

\section{BIBLIOGRAPHIE}

Arnold, J. (2006). « Comment les facteurs affectifs influencent-ils l'apprentissage d'une langue étrangère?». Ela, vol. 4, pp. 407-425.

Austin, J. L. (1962). How to do things with words. Oxford: Oxford University Press.

Behra, S. \& Macaire, D. (2017). « Souvenirs et croyances sur les langues et leur apprentissage chez des futurs enseignants ». Études en didactique des langues, vol. 28, pp. 115-136.

Bourguignon, C. (2011). « L'apprentissage des langues par l'action ». In Lions-Olivieri, M.-L. \& Liria, P. (dir.). L'approche actionnelle dans l'enseignement des langues : douze articles pour mieux comprendre et faire le point. Barcelone : Difusión \& Paris : Maison des langues.

Carvalho, D. P. (2015). « L'observation et l'enregistrement des cours de langue des signes française comme langue étrangère ». SHS Web of Conferences, vol. 20. 
Décuré, N. (2000). « Je suis timide et je n'ai rien à dire mais je me soigne. Résultats d'une enquête sur l'expression orale ». Les Après-midi de LAIRDIL, vol. 11, pp. 33-50.

Décuré, N., Lavinal, F. \& Blois, A. (2005). « La motivation, vecteur indispensable dans l'apprentissage des langues ». Passerelle, nº spécial. Oran : Dar El Gharb, pp. 195-212.

Dörnyei, Z. \& Kormos, J. (1998). « Problem-solving mechanisms in 12 communication. A Psycholinguistic Perspective ». Studies in Second Language Acquisition, vol. 20, n 3. pp. 349-385.

Droit-Volet, Sylvie. (2005). « Le long apprentissage du temps ». Pour la science, 328, pp. 2-7.

Figueiredo, E. H. D. de \& Bota, M. B. (2009). «L2 speech production in the classroom: the effects of teaching speaking in its own right ». Revista Intercâmbio, vol. 19, pp. 101-124.

Gan, Z. (2012). « Understanding 12 speaking problems: implications for ESL curriculum development in a teacher training institution in Hong Kong ». Australian Journal of Teacher Education, vol. 37, $\mathrm{n}^{\circ} 1$. pp. 43-59.

Germain, C. \& Netten, J. (2005). « Place et rôle de l'oral dans l'enseignement/apprentissage d'une L2 ». Babylonia, vol. 2, pp. 7-10.

Hilton, H. (2011). « Psycholinguistique de la production orale, aisance et disfluence en L2 ». In Chini, D. \& Goutéraux, P. Intégration de l'altérité dans l'apprentissage des langues : formes didactiques et procédures psycholinguistiques. Paris: L'Harmattan, pp. 75-96.

Kern, R. G. (1995). « Students' and teachers' beliefs about language learning ». Foreign Language Annals, vol. 28, $n^{\circ}$ 1. pp. 71-92.

Moussa, L. (2010). « An investigation of social interaction in the second language learning process: an alternate approach to second language pedagogy in Greece ». http:// arts.brighton.ac.uk/__data/assets/pdf_file/0004/19741/TLM22-Second-Language-Acquisition MA-TESOL-University-of-Brighton-Laura-Moussa.pdf.

Nguyen, T. C. (2014). Du rituel communicatif en classe de langue au rituel de la communication verbale quotidienne : prise de conscience de ce passage chez les étudiants de français à l'université de Cantho. Thèse de doctorat de l'université Paul-Valéry - Montpellier 3.

Osboe, S., Fujimura, T. \& Hirschel, R. (2007). « Student confidence and anxiety in L2 speaking activities ». Proceedings of the Independent Learning Association 2007 Japan Conference: Exploring theory, enhancing practice: Autonomy across the disciplines. Kanda University of International Studies, Chiba, Japan, October 2007. https://www.independentlearning.org/ uploads/100836/ILA2007_032.pdf (consulté le 23/2/2017).

Park, H. \& Lee A. R. (2007). « L2 learners' anxiety, self-confidence and oral performance ». Proceedings of the $10^{\text {th }}$ Conference of Pan-Pacific Association of Applied Linguistics, pp. 197-208 Pogranova, S., Brauchli B. \& Elmiger D. (2017). «Représentations sociales de la réussite d'une leçon de langue ». Études en didactique des langues, vol. 28, pp. 97-112.

Snauwaert, F. (2015). « Combien d'heures faut-il pour apprendre l'anglais? » BilingueAnglais.com. https://bilingueanglais.com/blog/1/combien-d-heures-faut-il-pour-apprendre-l-anglais/).

Spratt, M. (1999). « How good are we at knowing what learners like?» System, vol. 27, $\mathrm{n}^{\circ} 2$. pp. 141-155.

Tighilet, S. (2016). La communication en classe de langue. Une analyse comparative de la volonté de communiquer et de l'utilisation des stratégies de communication dans un contexte LANSAD en Algérie et en France. Thèse de doctorat, Université de Toulouse. 
Weissheimer, J. \& Borges Mota, M. (2011). « Working memory capacity and the development of L2 speech production: a study of individual differences ». In Granena, G. et al. (Ed.). Selected Proceedings of the 2010 Second Language Research Forum, pp. 169-181.

Yoshimura, Y. \& MacWhinney, B. (2007). « The effect of oral repetition on L2 speech fluency: an experimental tool and language tutor ». SLaTE-2007, pp. 25-28.

\section{ANNEXES}

\section{Annexe 1 - Le questionnaire étudiant}

L'oral

Définition

Dire quelque chose en anglais.

Toutes les activités sont concernées : poser une question ou y répondre, exposés, débats, jeux (de rôles), balbutiements, lecture à haute voix, etc.

Avez-vous parlé anglais pendant ce cours?

1a $\square$ Non. Pourquoi?

$1 \mathrm{~b} \square$ Oui. Moins d'une minute

1c $\square$ Oui. Entre 1 et 2 minutes

$1 \mathrm{~d} \square$ Oui. De 2 à 5 minutes

1e $\square$ Oui. Entre 5 et 15 minutes

1f $\square$ Oui. Plus de 15 minutes

À qui ?

2a $\square$ La/le prof (seul-e)

$2 b \square$ D'autres étudiant/es (à 2 ou en groupes)

2c $\square$ Toute la classe (prof + étudiant/es)

Avez-vous eu des difficultés?

3a $\square$ Non.

$3 \mathrm{~b} \square$ Oui. Manque de vocabulaire

3c $\square$ Oui. Problèmes de prononciation

$3 \mathrm{~d} \square$ Oui. Problèmes de grammaire

3e $\square$ Oui. Difficulté à comprendre les autres

3f $\square$ Oui. Manque d'idées

3g $\square$ Oui. Timidité / angoisse / inhibition

3h $\square$ Oui. Manque de motivation / d'envie / découragement

Je suis satisfait.e $\square$ / Je ne suis pas satisfait.e $\square$ parce que :

$4 \mathrm{a} \square$ Je n'ai pas parlé ou peu parlé

$4 \mathrm{~b} \square$ J'ai suffisamment parlé

$4 \mathrm{c} \square$ J'ai beaucoup/trop parlé

\section{Annexe 2 - Le carnet de bord}

\begin{tabular}{|l|l|l|l|l|l|l|l|l|}
\hline Prof. & Groupe & Niveau & Date & Jour & Horaire & Effectif & $\mathrm{Nb} H / \mathrm{F}$ & Séance ${ }^{\circ}$ \\
\hline
\end{tabular}




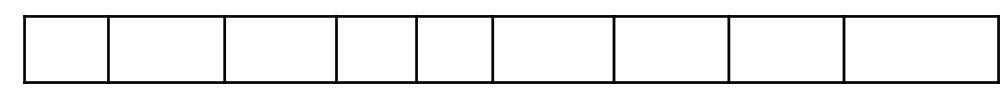

Durée de l'activité

$10 \mathrm{mn}$

$20 \mathrm{mn}$

$30 \mathrm{mn}$

$45 \mathrm{mn}$

1 hoo

$1 \mathrm{~h} 15$

$1 \mathrm{~h} 30$

$1 \mathrm{~h} 45$

$2 \mathrm{~h} 00$

Topographie de la classe

en $\mathrm{U}$

en rangs d'oignons

en groupes autour d'une (grande) table

autre.........

Prise de parole préparée

avant le cours avant l'exercice

avec input

en groupe

individuellement

Expression guidée

réponse à questions prof

en laboratoire

dialogues

lecture à haute voix

Expression libre

réaction à docs présentés

jeux de rôles

débats

dialogues

Configuration avec la classe entière

chacun/e parle à tour de rôle

chacun/e parle quand il/elle le sent

chacun/e répond quand on l'interroge

Configuration en groupes

paires

groupes de 3-4

groupes de 5-6

tête-à-tête avec prof (le reste fait autre chose) 


\section{Annexe 3 - Les activités}

\begin{tabular}{|c|c|c|c|c|}
\hline $\begin{array}{l}\text { Type d'activité } \\
\text { Activité }\end{array}$ & $\begin{array}{l}\text { Parole guidée, } \\
\text { contrainte/ } \\
\text { spontanée }\end{array}$ & $\begin{array}{l}\text { Avec ou } \\
\text { sans } \\
\text { support }\end{array}$ & $\mid \begin{array}{l}\text { Classe entière/ W } \\
\text { à deux/ W en } \\
\text { petit groupe }\end{array}$ & $\begin{array}{l}\text { Préparation maison } \\
\text { ou cours }(\mathrm{O}) \text { ou rien } \\
(\mathrm{N})\end{array}$ \\
\hline \multicolumn{5}{|l|}{ JEUX } \\
\hline Tabou & 2 & 0 & GC & $\mathrm{N}$ \\
\hline Jeu des 7 familles & 3 & 0 & G & $\mathrm{N}$ \\
\hline Flash cards & 3 & 0 & C & $\mathrm{N}$ \\
\hline $\begin{array}{l}\text { Épreuves (How much } \\
\text { will you bet?) }\end{array}$ & 2 & 0 & G & $\mathrm{N}$ \\
\hline It's in the cards & 2 & 0 & G & $\mathrm{N}$ \\
\hline Trivial pursuit & 3 & 0 & GC & $\mathrm{N}$ \\
\hline Jeopardy & 3 & 0 & G & $\mathrm{N}$ \\
\hline Jeux de vocabulaire & 3 & 0 & GC & $\mathrm{N}$ \\
\hline Morpion & 3 & 0 & G & $\mathrm{N}$ \\
\hline Mimes & 3 & 0 & GC & $\mathrm{N}$ \\
\hline Quiz & 3 & 0 & GC & $\mathrm{N}$ \\
\hline Drills & 3 & 0 & GC & $\mathrm{N}$ \\
\hline Jeux type monopoly & 3 & 0 & G & $\mathrm{N}$ \\
\hline Snakes and ladders & 3 & 0 & G & $\mathrm{N}$ \\
\hline Find someone who & 3 & 0 & GC & $\mathrm{N}$ \\
\hline \multicolumn{5}{|l|}{ DÉBATS } \\
\hline A l'anglaise & 1 & $\mathrm{O} / \mathrm{N}$ & C & $\mathrm{O} / \mathrm{N}$ \\
\hline Issues & 1 & $\mathrm{O} / \mathrm{N}$ & GC & $\mathrm{O} / \mathrm{N}$ \\
\hline \multicolumn{5}{|c|}{ EXPOSÉS / PRÉSENTATIONS } \\
\hline $\begin{array}{l}\text { Présentation } \\
\text { personnelle }\end{array}$ & 1 & $\mathrm{~N}$ & GC & $\mathrm{N}$ \\
\hline $\begin{array}{l}\text { Exposé (court ou } \\
\text { long) }\end{array}$ & 1 & 0 & C & 0 \\
\hline
\end{tabular}




\begin{tabular}{|c|c|c|c|c|}
\hline $\begin{array}{l}\text { Présentations de } \\
\text { nature } \\
\text { professionnelle }\end{array}$ & 1 & 0 & GC & $\mathrm{O} / \mathrm{N}$ \\
\hline \multicolumn{5}{|c|}{ LECTURE A VOIX HAUTE / PRONONCIATION } \\
\hline Poème & 3 & 0 & $G$ & $\mathrm{~N}$ \\
\hline $\begin{array}{l}\text { Définitions/ } \\
\text { vocabulaire }\end{array}$ & 3 & 0 & GC & $\mathrm{N}$ \\
\hline Dictée & 3 & 0 & GC & $\mathrm{N}$ \\
\hline Chanter & 3 & 0 & C & $\mathrm{N}$ \\
\hline Tongue twisters & 3 & 0 & GC & $\mathrm{N}$ \\
\hline \multicolumn{5}{|l|}{ REFORMULATION } \\
\hline Définitions & 3 & 0 & C & $\mathrm{N}$ \\
\hline Résumé & 2 & 0 & GC & $\mathrm{O} / \mathrm{N}$ \\
\hline $\begin{array}{l}\text { Information } \\
\text { lacunaire }\end{array}$ & 3 & 0 & G & $\mathrm{N}$ \\
\hline Compléter notes & 3 & 0 & GC & $\mathrm{N}$ \\
\hline $\begin{array}{l}\text { Questions pour } \\
\text { comprendre }\end{array}$ & 3 & 0 & GC & $\mathrm{N}$ \\
\hline \multicolumn{5}{|l|}{ IMPROVISATION } \\
\hline Sketches & 1 & 0 & $G$ & $\mathrm{O} / \mathrm{N}$ \\
\hline Brainstorming & 1 & $\mathrm{O} / \mathrm{N}$ & GC & $\mathrm{N}$ \\
\hline $\begin{array}{l}\text { Coup de cœur/coup } \\
\text { de gueule }\end{array}$ & 1 & $\mathrm{~N}$ & C & $\mathrm{N}$ \\
\hline Best/worst & 1 & $\mathrm{~N}$ & C & $\mathrm{N}$ \\
\hline $\begin{array}{l}\text { One minute to } \\
\text { convince }\end{array}$ & 1 & $\mathrm{~N}$ & GC & $\mathrm{N}$ \\
\hline $\begin{array}{l}\text { Jeux de rôles/ } \\
\text { simulations }\end{array}$ & 1 & 0 & GC & $\mathrm{N}$ \\
\hline Décrire image & 1 & 0 & $G$ & $\mathrm{~N}$ \\
\hline Sujets personnels & 1 & $\mathrm{~N}$ & CG & $\mathrm{N}$ \\
\hline Topics & 1 & $\mathrm{O} / \mathrm{N}$ & $G$ & $\mathrm{~N}$ \\
\hline
\end{tabular}




\begin{tabular}{|l|l|l|l|l|}
\hline $\begin{array}{l}\text { Dialogues } \\
\text { idiomatiques }\end{array}$ & 2 & O & G & O \\
\hline Narration & 1 & O & G & N \\
\hline
\end{tabular}

Contrainte (1 à 3 : du moins au plus contraint)

Toute activité possède un degré de contrainte car imposée, en général, par l'enseignante, qui guide en donnant à la fois le cadre, le format et souvent le sujet. Dans beaucoup d'activités, l'étudiant.e peut choisir de ne pas prendre la parole ou s'en tenir au minimum. Il/elle peut aussi choisir de parler spontanément, souvent, longtemps.

\section{Configuration}

$G$ : Travail en groupe de 2 à 6.

$C$ : Travail effectué avec la classe entière.

Dans les groupes de A, le travail à deux ou en groupes ne fait pas l'objet d'une étape collective avec toute la classe en fin d'activité. Dans les groupes d'B, toutes les activités de groupe ont une phase finale collective.

Support : cela peut aller d'un mot (sujet d'une impro) à un texte entier.

Préparation

Seuls les exposés et les présentations se préparent à la maison.

\section{Annexe 4- Le questionnaire-bilan}

\begin{tabular}{|c|c|c|}
\hline 1 & Avez-vous rempli les questionnaires avec sérieux? & $\begin{array}{l}\square \text { Oui } \\
\square \text { Non }\end{array}$ \\
\hline 2 & $\begin{array}{l}\text { Dans laquelle/lesquelles de ces propositions vous reconnaissez- } \\
\text { vous? } \\
\text { Entourez : } \\
1 \text { A - } 1 \text { B - } 1 \text { C - 2A - } 2 B-2 C \\
1 \square \text { Je suis satisfait.e } \\
2 \square \text { Je ne suis pas satisfait.e }\end{array}$ & $\begin{array}{l}A \square \text { d'avoir peu ou pas } \\
\text { parlé } \\
B \square \text { d'avoir beaucoup } \\
\text { parlé } \\
C \square \text { d'avoir trop parlé }\end{array}$ \\
\hline 3 & $\begin{array}{l}\text { Ce questionnaire vous a-t-il aidé.e à prendre conscience de votre } \\
\text { participation à l'oral? }\end{array}$ & $\begin{array}{l}\square \text { Oui } \\
\square \text { Non }\end{array}$ \\
\hline 4 & $\begin{array}{l}\text { Ce questionnaire vous a-t-il aidé.e à prendre conscience de vos } \\
\text { difficultés à l'oral? }\end{array}$ & $\begin{array}{l}\square \text { Oui } \\
\square \text { Non }\end{array}$ \\
\hline 5 & Avez-vous perçu cette enquête comme une contrainte? & $\begin{array}{l}\square \text { Oui } \\
\square \text { Non }\end{array}$ \\
\hline 6 & Avez-vous aimé y participer? & $\begin{array}{l}\square \text { Oui } \square \text { Indifférent } \\
\square \text { Non }\end{array}$ \\
\hline 7 & $\begin{array}{l}\text { Avez-vous trouvé cette enquête utile pour développer la pratique } \\
\text { de l'oral en classe? }\end{array}$ & $\begin{array}{l}\square \text { Oui } \\
\square \text { Non }\end{array}$ \\
\hline 8 & Avez-vous des remarques? & \\
\hline
\end{tabular}




\section{NOTES}

1. SID : Statistiques et informatique décisionnelle ; BCP : Biologie cellulaire et physiologie ; MSE : Modélisation des systèmes écologiques.

2. SID : Statistiques et informatique décisionnelle ; BCP : Biologie cellulaire et physiologie ; MSE : Modélisation des systèmes écologiques.

\section{RÉSUMÉS}

L'expression orale en anglais est maintenant, au centre des préoccupations des enseignant.e.s, y compris dans le secteur LANSAD qui, dans ce domaine précis, reste encore largement inexploré. Dans cet article, nous présentons les résultats d'une enquête fondée sur un questionnaire soumis à un échantillon d'étudiants dans le secteur LANSAD-sciences. L'objectif de cette recherche était d'évaluer le temps de parole de chaque étudiant.e pour obtenir une meilleure image de ce qui se passe réellement dans une classe censée être centrée sur l'oral. Elle vise à cerner les facteurs, à la fois positifs et négatifs, qui influent sur la production orale afin de proposer des pistes pour améliorer la prise de parole.

Oral expression in English is now at the center of teachers' concerns, including in the ESP sector which is still, on this particular issue, fairly unexplored. In this paper, we present the results of a survey based on a questionnaire submitted to a sample of science students in ESP courses. The aim of this research was to evaluate each student's speech production to get a better picture of what is actually happening in a class that is supposed to be focused on oral skills. The study identifies both positive and negative factors influencing oral production in order to suggest remedial means of improving the situation.

\section{INDEX}

Keywords : speaking, ESP, task-based learning, time awareness

Mots-clés : prise de parole, LANSAD, activités pédagogiques, perception du temps

\section{AUTEURS}

\section{ELISABETH CROSNIER}

Elisabeth Crosnier est MCF (retraitée) à l'Université Toulouse $3\left(11^{\mathrm{e}}\right.$ section du CNU). Le cadre théorique et méthodologique de ses recherches est celui de l'anglais de spécialité et de la didactique des langues en secteur LANSAD. Ses recherches ont porté sur la connaissance d'une communauté, de ses problèmes et de ses besoins langagiers : l'analyse de discours scientifique, la motivation en classe de langue, l'autonomie, le processus d'acquisition et d'apprentissage de l'anglais langue étrangère à l'université ; l'aide à la rédaction en anglais scientifique ; la 
communication orale en anglais en milieu professionnel ; l'approche contrastive et la traduction français/anglais ; l'interculturalité ; la didactique de l'anglais à vocation professionnelle dans la filière LEA.

elisabeth.crosnier@orange.fr

\section{NICOLE DÉCURÉ}

Nicole Décuré est professeure émérite à l'Université Toulouse $3\left(11^{\mathrm{e}}\right.$ section du $\left.\mathrm{CNU}\right)$. Elle est rédactrice en chef des revues Études en didactique des langues (EDL) et Pédagogie des langues étrangères (PLE). Ses travaux de recherche en didactique LANSAD portent sur le jeu, la motivation, les compétences langagières. Elle est aussi spécialiste de littérature féministe, notamment le roman policier.

nicdec@orange.fr 